\title{
Editorial of special issue on ocean transportation logistics: making global supply chain effective
}

\author{
Kjetil Fagerholt $^{1}$ - Kap-Hwan Kim ${ }^{2}$. \\ Chung-Yee Lee ${ }^{3}$ Q Qiang Meng ${ }^{4}$ Xiangtong $\mathbf{Q i}^{3}$
}

Maritime logistics plays an important role in enabling global supply chain networks. The research of maritime logistics has expanded its scope, from terminal operations and vessel routing, to strategic-level problems such as competition and collaboration issues. This special issue of the Flexible Services and Manufacturing (FSM) Journal aims to address problems related to maritime logistics from various perspectives.

The idea of editing this special issue was initiated with the planning of The 5th International Conference on Logistics and Maritime Systems (LOGMS 2015). The conference, sponsored by the Theme-based Research Scheme of Hong Kong RGC (T32-620/11), was held in The Hong Kong University of Science and Technology, 27-29 August 2015. We promoted this special issue to the conference participants,

Chung-Yee Lee
cylee@ust.hk
Kjetil Fagerholt
kjetil.fagerholt@iot.ntnu.no

Kap-Hwan Kim

kapkim@pusan.ac.kr

Qiang Meng

ceemq@nus.edu.sg

Xiangtong Qi

ieemqi@ust.hk

1 Department of Industrial Economics and Technology Management, Department of Marine Technology, Norwegian University of Science and Technology, Trondheim, Norway

2 Department of Industrial Engineering, Pusan National University, Pusan, Korea

3 Department of Industrial Engineering and Logistics Management, The Hong Kong University of Science and Technology, Clear Water Bay, Hong Kong SAR

4 Department of Civil and Environmental Engineering, National University of Singapore, Singapore, Singapore 
and at the same time, we opened the submission to all researchers interested in maritime logistics. We received 30 submissions in total. After rigorous reviews, 11 papers were accepted. These papers cover a broad range of maritime logistics, which can be classified into three areas.

There are five papers studying container terminal operations, a conventional area with new issues to be addressed. Yu et al. (2017) study a quay crane scheduling problem which considers the tidal impact. Petering et al. (2017) study a problem of container storage location assignment at a transshipment Terminal. Two other papers consider the benefit of in-advance booking or making appointments, Gracia et al. (2017) aiming to reduce the terminal gate congestion, and Covic (2017) investigating how to facilitate the container re-marshalling operations. While the above consider problems for a single terminal, Heilig et al. (2017), study a problem of truck routing involving terminals and nearby inland facility sites.

Besides terminal operations, vessel routing is another important area of maritime logistics. There are two papers falling into this area. Thun et al. (2017) study liner network design problem with complex services structures, and Tirado and Hvattum (2017) investigate the problem of determining vessel departure time at a port in a given route.

The last area is about problems beyond the conventional scope of maritime logistics. There are two papers addressing supply chain management involving ocean transportation, Andersen et al. (2017) focusing on the distribution of wood pallets, and Fan et al. (2017) proposing a general supply chain model. Revenue management, which has been well adopted in airlines, is less explored in the industry of maritime logistics. This special issue has one paper in this area, van Riessen et al. (2017), studying the pricing problem for different cargo classes. Finally, Yu et al. (2017) study the port-to-port competition in one region by gametheoretic analysis.

This special issue is the outcome from the joint hard work of the authors, reviewers, and the editorial team. We would like to thank all people involved in the process, as well as Hong Kong RGC Theme-based Research Scheme (T32-620/11) for the support to LOGMS 2015 and this special issue.

Kjetil Fagerholt is a Professor in Operations Research at the Norwegian University of Science and Technology, Department of Industrial Economics and Technology Management. He also holds a position as an Adjunct Professor at the Department of Marine Technology at the same university, and as a Scientific Adviser at SINTEF Ocean. His main research interest is on developing and applying Operations Research models and methods on planning problems in maritime transportation. He has published about 90 papers in international peer-reviewed journals, such as Transportation Science, European Journal of Operational Research, OMEGA, Transportation Research Part C, D and E, INFORMS Journal on Computing, Computers \& Operations Research and Journal of the Operational Research Society. He is Associate Editor for Transportation Science and Asia-Pacific Journal of Operational Research, and Deputy Editor for Journal of Marine Science and Technology.

Kap-Hwan Kim working at the Department of Industrial Engineering of Pusan National University. He studied at the Seoul National University (Bachelor) and the Korea Advanced Institute of Science and Technology (Master, Ph.D.). He was a visiting scholar at Purdue University, Montreal University, and Shanghai Maritime University. He was the director of the Institute of Logistics Innovation and 
Networking at Pusan National University and the president of the Korean Institute of Industrial Engineers. His research is focused on the design and operational problems of container terminals. He published many papers at international journals such as Flexible Services and Manufacturing Journal, Transportation Science, Transportation Research B, Transportation Research E, European journal of Operational Research, and so on.

Chung-Yee Lee is currently Director of the Office of Institutional Research at Hong Kong University of Science and Technology (HKUST). He is also Senior Advisor to the Vice-President for Research \& Graduate Studies, and Visiting Professor of Industrial Engineering \& Logistics Management Department at HKUST. He is a Fellow of the Institute of Industrial Engineers in U.S. and also a Fellow of Hong Kong Academy of Engineering Sciences. Before joining HKUST in 2001, he was Rockwell Chair Professor in the Department of Industrial Engineering at Texas A \& M University. His research interests are in logistics and supply chain management, scheduling and inventory management, with a current focus on ocean transport logistics and e-commerce logistics. He has published more than 160 papers in refereed journals.

Qiang Meng is a professor in Department of Civil and Environmental Engineering at National University of Singapore. He received his Ph.D. from Department of Civil and Environmental Engineering at The Hong Kong University of Science and Technology in 2000. His research focuses include transportation network modeling and optimization, shipping and intermodal freight transportation analysis, and quantitative risk assessment of transport operations. He has clinched a number of research awards and prizes, including Changjiang Scholar Chair Professor appointed by Ministry of Education of PR China in 2017, Outstanding Alumni Award of Department of Civil and Environmental Engineering at The Hong Kong University of Science and Technology in 2016 and Dean's Chair in Faculty of Engineering at National University of Singapore in 2015.

Xiangtong Qi is a professor at the Department of Industrial Engineering and Logistics Management, Hong Kong University of Science and Technology. His main research interest is optimization and game theoretic analysis, with applications in production scheduling, inventory control, logistics and supply chain management. He has published more than fifty papers in major academic journals, and co-authored the book Disruption Management: Framework, Models and Applications. He has been as Associate Editor for the journals of IIE Transactions, IEEE Transactions on Automation Science and Engineering, and Asia-Pacific Journal of Operations Research. 